CARPATHIAN J. MATH.

Volume 38 (2022), No. 2,

Pages $281-297$

\title{
An accelerated Visco-Cesaro means Tseng Type splitting method for fixed point and monotone inclusion problems
}

\author{
Yasir Arfat $^{1}$, POOM Kumam ${ }^{1,2,3}$, Muhammad Aqeel Ahmad KHAN ${ }^{4}$ and Parinya \\ SA NGIAMSUNTHORN ${ }^{5}$
}

\begin{abstract}
In this paper, we study a variant of Tseng's splitting method for monotone inclusion problem and fixed point problem associated with a finite family of $\eta$-demimetric mappings in Hilbert spaces. The proposed algorithm is based on the combination of classical Tseng's method together with the viscosity Cesáro means method and the Nesterov's acceleration method. The proposed iterative method exhibits accelerated strong convergence characteristics under suitable set of control conditions in such framework. Finally, we provide a numerical example to illustrate the applicability of the proposed algorithm as well as some useful abstract applications.
\end{abstract}

\section{INTRODUCTION AND PRELIMINARIES}

Let $\mathcal{H}$ be a real Hilbert space with the inner product $\langle\cdot, \cdot\rangle$ and the associated norm $\|\cdot\|$. The classical monotone inclusion problem aims to find

$$
x^{*} \in \mathcal{H} \text { such that } 0 \in A x^{*}+B x^{*},
$$

where $A: \mathcal{H} \rightarrow 2^{\mathcal{H}}$ is a multi-valued operator and $B: \mathcal{H} \rightarrow \mathcal{H}$ is a single-valued operator.

The problem (1.1), in the context of monotone operator theory, has been largely considered for modeling various real world as well as theoretical problems in the field of convex optimization, subgradients, partial differential equations, variational inequalities and image processing, evolution equations and inclusions, see for instance, $[15,14,17,32]$ and the references cited therein.

Since (1.1) is complex in nature and therefore requires sophisticated tools and iterative algorithms for the consequent analysis. The elegant forward-backward (FB) iterative algorithm [27, 30] is prominent among various iterative algorithms to solve (1.1). It is worth mentioning that FB iterative algorithm exhibits weak convergence even assuming the stronger conditions on the operators $A$ and $B$. Later, Tseng [35] modified the FB iterative algorithm for weak convergence results in Hilbert spaces. Recently, Gibali and Thong [21] considered a modified variant of the Tseng's splitting method to establish strong convergence results in Hilbert spaces. We remark that the several general splitting algorithms are available in the literature with specific limitations. However, new splitting algorithms are formulated in such a way to unify and/or combine the existing splitting algorithms with enhanced intrinsic properties. We, therefore, propose and analyze a splitting method comprises of forward-backward-forward (FBF) iterates or Tseng's splitting method in Hilbert spaces.

We now elaborate some necessary concepts of fixed point theory as follows:

Received: 27.04.2021. In revised form: 28.10.2021. Accepted: 06.11.2021

2010 Mathematics Subject Classification. 47H05, 47H06, 47H09, 47H10, 65Y05, 65K15, 68W10.

Key words and phrases. monotone inclusion problem, forward-backward-forward method, viscosity approximation method, Cesáro means method, Nesterov's accelerated method, strong convergence.

Corresponding author: Poom Kumam; poom.kum@kmutt.ac.th 
Let $C$ be a nonempty subset of a real Hilbert space $\mathcal{H}$. For an operator $S: C \rightarrow C$, the set Fix $(S)=\{x \in C \mid x=S x\}$ denotes the set of fixed points of the operator $S$. Recall that the operator $S$ is called (i) nonexpansive, if $\|S x-S y\| \leq\|x-y\|$, for all $x, y \in C$; (ii) quasi-nonexpansive, if $\operatorname{Fix}(S) \neq \emptyset$ and $\|S x-y\| \leq\|x-y\|$, for all $x \in C$ and $y \in$ Fix $(S)$; (iii) firmly nonexpansive if for each $x, y \in \mathcal{H}$ such that $\|S x-S y\|^{2} \leq\langle S x-S y, x-y\rangle$ (iv) $\eta$-strict pseudocontraction [11], if there exists $\eta \in[0,1)$ such that

$$
\|S x-S y\|^{2} \leq\|x-y\|^{2}+\eta\|x-y-(S x-S y)\|^{2}, \text { for all } x, y \in C ;
$$

(v) $\eta$-demicontractive if $\operatorname{Fix}(S) \neq \emptyset$ and there exists $\eta \in[0,1)$ such that

$$
\|S x-y\|^{2} \leq\|x-y\|^{2}+\eta\|x-S x\|^{2} \text {, for all } x \in C \text { and } y \in \operatorname{Fix}(S) ;
$$

(vi) $\eta$-demimetric [33] where $\eta \in(-\infty, 1)$, if $\operatorname{Fix}(S) \neq \emptyset$ such that

$$
\langle x-y,(I d-S) x\rangle \geq \frac{1}{2}(1-\eta)\|(I d-S) x\|^{2}, \text { for all } x \in C \text { and } y \in \operatorname{Fix}(S),
$$

where $I d$ denotes the identity operator. Note that

$$
\|S x-y\|^{2} \leq\|x-y\|^{2}+\eta\|x-S x\|^{2} \text {, for all } x \in C \text { and } y \in F i x(S) .
$$

is an equivalent representation of an $\eta$-demimetric operator. It is evident that the class of $\eta$-demimetric operators contains the operators defined in (i)-(iv).

Fixed point theory of nonlinear operators is a fertile field of research and emerged as a powerful tool to solve a variety of problems arising in various branches of sciences [15, 22, 23]. In 1975, Baillon[9] established the first nonlinear ergodic theorem as follows:

Theorem 1.1 ([9]). Let $C$ be a nonempty, closed and convex subset of a real Hilbert space $\mathcal{H}$ and let $S: C \rightarrow C$ be a nonexpansive operator such that $F i x(S) \neq \emptyset$ then for all $x \in C$, the Cesáro means

$$
S_{n} x=\frac{1}{N} \sum_{i=0}^{N-1} S_{i} x, \forall N \geq 1,
$$

weakly converges to a fixed point of $S$.

Since then the classical Cesáro means method have been considered for various classes of nonlinear operators, see $[16,25,26]$ and the references cited therein. It is worth mentioning that the Cesáro means method fails to converge strongly for the class of nonexpansive operators[20]. In order to obtain strong convergence results, one has to impose additional requirements on the iterative algorithm. In 1967, Halpern[24] introduced and analyzed an iterative algorithm which strongly converges to the closest fixed point of the nonexpansive operator. It is remarked that the Halpern iterative algorithm coincides with the Cesáro means method for linear operators. In 2000, Moudafi [29] proposed and analyzed the strongly convergent viscosity iterative algorithm by utilizing a strict contraction operator instead of the anchor point in the Halpern iterative algorithm. In this paper, we are going to study an algorithm based on the combination of classical Tseng's method associated with the monotone inclusion problem together with the viscosity Cesáro means method for $\eta$-demimetric operators. In order to enhance the speed of convergence of the proposed iterative algorithm, we also utilize the inertial extrapolation technique essentially due to Polyak [31], see also[1, 2, 3, 4, 5, 6, 7].

The rest of the paper is organized as follows: Section 2 contains preliminary concepts and results regarding monotone operator theory and fixed point problem theory. Section 3 comprises strong convergence results of the proposed algorithm whereas Section 4 deals with the efficiency of the proposed algorithm and its comparison with the existing algorithm by numerical experiments. Section 5 provides various abstract applications of of 
the proposed algorithm in minimization problems, split feasibility problems and image processing.

\section{PRELIMinaries}

We start this section with the mathematical preliminary concepts required in the sequel. An operator $P_{C}^{\mathcal{H}}$ is said to be metric projection of $\mathcal{H}$ onto nonempty, closed and convex subset $C$ if for every $x \in \mathcal{H}$, there exists a unique nearest point in $C$ denoted by $P_{C}^{\mathcal{H}} x$ such that

$$
\left\|x-P_{C}^{\mathcal{H}} x\right\| \leq\|x-z\|, \text { for all } z \in C .
$$

It is remarked that the metric projection operator satisfies firmly nonexpansiveness and can be characterized as:

$$
\left\langle x-P_{C}^{\mathcal{H}} x, P_{C}^{\mathcal{H}} x-y\right\rangle \geq 0, \text { for all } x \in \mathcal{H} \text { and } y \in C .
$$

Recall that a set-valued operator $A: \mathcal{H} \rightarrow 2^{\mathcal{H}}$ is called monotone, if for all $x, y \in \mathcal{H}$, $u \in A x$ and $v \in A y$ then $\langle x-y, u-v\rangle \geq 0$. Moreover, a monotone operator $A$ is said to be maximal monotone if there is no proper monotone extension of $A$. For a monotone operator $A$, the associated resolvent operator $J_{m}^{A}$ of index $m>0$ is defined as

$$
J_{m}^{A}=(I d+m A)^{-1},
$$

where $(\cdot)^{-1}$ denotes the inverse operator.

Note that the resolvent operator $J_{m}^{A}$ is well defined everywhere on Hilbert space $\mathcal{H}$. Further, $J_{m}^{A}$ is single valued and satisfies the firmly nonexpansiveness. Furthermore, $x \in$ $A^{-1}(0)$ if and only if $x=J_{m}^{A}(x)$.

Definition 2.1. Let $S: C \rightarrow C$ be a nonexpansive operator defined on a nonempty, closed and convex subset of a real Hilbert space $\mathcal{H}$. The operator $I d-S$ is said to be demiclosed at the origin provided that for any sequence $\left(x_{n}\right)$ in $C$ that converges weakly to some $x$ and if the sequence $\left((I d-S) x_{n}\right)$ converges strongly to 0 , then $(I d-S)(x)=0$.

The rest of this section is organized with the celebrated results required in the sequel.

Lemma 2.1. Let $x, y, z \in \mathcal{H}$ and $\alpha, \beta, \gamma \in[0,1] \subset \mathbb{R}$ and $\alpha+\beta+\gamma=1$ then we have

(1) $\|x+y\|^{2} \leq\|x\|^{2}+2\langle y, x+y\rangle$;

(2) $\|\beta x+(1-\beta) y\|^{2}=\beta\|x\|^{2}+(1-\beta)\|y\|^{2}-\beta(1-\beta)\|x-y\|^{2}$.

(3) $\|\alpha x+\beta y+\gamma z\|^{2}=\alpha\|x\|^{2}+\beta\|y\|^{2}+\gamma\|z\|^{2}-\alpha \beta\|x-y\|^{2}-\alpha \gamma\|x-z\|^{2}-\beta \gamma\|y-z\|^{2}$

Lemma 2.2 ([33]). Let $C$ be a nonempty, closed and convex subset of a Hilbert space $\mathcal{H}$ and let $S: C \rightarrow \mathcal{H}$ be an $\eta$-demimetric operator with $\eta \in(-\infty, 1)$. Then Fix $(S)$ is closed and convex.

Lemma 2.3 ([34]). Let $C$ be a nonempty, closed and convex subset of a Hilbert space $\mathcal{H}$ and let $S: C \rightarrow \mathcal{H}$ be an $\eta$-demimetric operator with $\eta \in(-\infty, 1)$ and Fix $(S) \neq \emptyset$. Let $\gamma$ be a real number with $0<\gamma<1-\eta$ and set $L=(1-\gamma) I d+\gamma S$, then $L$ is a quasi-nonexpansive operator of $C$ into $\mathcal{H}$.

Lemma 2.4 ([12]). Let $C$ be a nonempty bounded closed convex subset of a uniformly convex Banach space and $S: C \rightarrow C$ be a nonexpansive operator. For each $x \in C$ and the Cesáro means $S_{n} x=\frac{1}{N} \sum_{i=0}^{N-1} S_{i} x$, then $\lim \sup _{n \rightarrow \infty}\left\|S_{n} x-S\left(S_{n} x\right)\right\|=0$.

Lemma 2.5 ([10]). Let $A: \mathcal{H} \rightarrow 2^{\mathcal{H}}$ be a maximal monotone operator and $B: \mathcal{H} \rightarrow \mathcal{H}$ be a Lipschitz continuous and monotone operator. Then the operator $A+B$ is a maximal monotone operator. 
Lemma 2.6 ([21]). Let $A: \mathcal{H} \rightarrow 2^{\mathcal{H}}$ be a maximal monotone operator and $B: \mathcal{H} \rightarrow \mathcal{H}$ be a mapping on $\mathcal{H}$. Define $S_{\mu}:=(I d+\mu A)^{-1}(I d-\mu B), \mu>0$. Then we have Fix $\left(S_{\mu}\right)=$ $(A+B)^{-1}(0)$, for all $\mu>0$.

Lemma 2.7 ([37]). Let $\left(b_{n}\right)$ be a sequences of nonnegative real numbers and there exists $n_{0} \in \mathbb{N}$ such that:

$$
b_{n+1} \leq\left(1-\psi_{n}\right) b_{n}+\psi_{n} c_{n}+d_{n}, \forall n \geq n_{0},
$$

where $\left(\psi_{n}\right) \subset(0,1)$ and $\left(c_{n}\right),\left(d_{n}\right)$ with the following conditions hold:

(I) $\sum_{n=1}^{\infty} \psi_{n}=\infty$;

(II) $\limsup _{n \rightarrow \infty} c_{n} \leq 0$;

(III) $\sum_{n=1}^{\infty} d_{n}<\infty, \forall 0 \leq d_{n}(0 \leq n)$,

then $\lim _{n \rightarrow \infty} b_{n}=0$.

Lemma 2.8 ([28]). Let $\left(q_{n}\right)$ be a sequence of nonnegative real numbers. Suppose that there is a subsequence $\left(q_{n_{j}}\right)$ of $\left(q_{n}\right)$ such that $q_{n_{j}}<q_{n_{j}+1}$ for all $j \in \mathbb{N}$, then there exists a nondecreasing sequence $\left(\varepsilon_{k}\right)$ of $\mathbb{N}$ such that $\lim _{k \rightarrow \infty} \varepsilon_{k}=\infty$ and satisfy the following properties such that:

$$
q_{\varepsilon_{k}} \leq q_{\varepsilon_{k}+1} \text { and } q_{k} \leq q_{\varepsilon_{k}+1}
$$

for some large number $k \in \mathbb{N}$. Thus, $\varepsilon_{k}$ is the largest number $n$ in the set $\{1,2, \cdots, k\}$ such that $q_{n}<q_{n+1}$.

\section{MAIN RESULTS}

In this section, we prove the following strong convergence result.

Theorem 3.2. Let $A: \mathcal{H} \rightarrow 2^{\mathcal{H}}$ be a maximally monotone operator and let $B: \mathcal{H} \rightarrow \mathcal{H}$ be a monotone and $\rho$-Lipschitz operator for some $\rho>0$ defined on a real Hilbert space $\mathcal{H}$. For all $i \in\{1,2, \cdots, N\}$, let $S_{i}: \mathcal{H} \rightarrow \mathcal{H}$ be a finite family of $\eta$-demimetric operators with $\eta \in(-\infty, 1)$ such that $I d-S_{i}$ is demiclosed at the origin and let $h: \mathcal{H} \rightarrow \mathcal{H}$ be a contraction mapping with constant $\lambda \in[0,1)$. Assume that $\Gamma=(A+B)^{-1}(0) \cap \bigcap_{i=1}^{N} F i x\left(S_{i}\right) \neq \emptyset, \mu_{1}>0, \sigma \in(0,1)$, $\left(\xi_{n}\right) \subset[0,1)$ and $\left(\alpha_{n}\right),\left(\beta_{n}\right)$ are sequences in $(0,1)$. For given $x_{0}, x_{1} \in \mathcal{H}$, let the iterative sequence $\left(x_{n}\right)$ be generated by

$$
\left\{\begin{array}{l}
u_{n}=x_{n}+\xi_{n}\left(x_{n}-x_{n-1}\right) \\
v_{n}=J_{\mu_{n}}^{A}\left(I d-\mu_{n} B\right) u_{n} \\
w_{n}=v_{n}-\mu_{n}\left(B v_{n}-B u_{n}\right) \\
x_{n+1}=\alpha_{n} h\left(x_{n}\right)+\left(1-\alpha_{n}-\beta_{n}\right) x_{n}+\beta_{n}\left(\frac{1}{N} \sum_{i=0}^{N-1}\left(\left(1-\gamma_{n}\right) I d+\gamma_{n} S_{i}\right)\right) w_{n}
\end{array}\right.
$$

Assume that the following step size rule

$$
\mu_{n+1}=\left\{\begin{array}{l}
\min \left\{\frac{\sigma\left\|u_{n}-v_{n}\right\|}{\left\|B u_{n}-B v_{n}\right\|}, \mu_{n}\right\}, \text { if } B u_{n}-B v_{n} \neq 0 \\
\mu_{n}, \text { otherwise, }
\end{array}\right.
$$

and the conditions hold:

(C1) $\sum_{n=1}^{\infty} \xi_{n}\left\|x_{n}-x_{n-1}\right\|<\infty$;

(C2) $\lim _{n \rightarrow \infty} \alpha_{n}=0$ and $\sum_{n=1}^{\infty} \alpha_{n}=\infty$, and for each $n \in \mathbb{N}, 0<a^{*}<\liminf _{n \rightarrow \infty} \beta_{n} \leq$ $\limsup _{n \rightarrow \infty} \beta_{n}<b^{*}<1-\alpha_{n}$, where $a^{*}, b^{*}$ be positive real numbers.

Then the sequence $\left(x_{n}\right)$ generated by (3.2) converge strongly to a point $\bar{x}=P_{\Gamma} \circ h(\bar{x})$.

In order to prove Theorem 3.2, we need the following results from [21].

Lemma 3.9 ([21]). The sequence $\left(\mu_{n}\right)$ generated by (3.2) is a nonincreasing sequence with a lower bound of $\min \left\{\mu_{1}, \frac{\sigma}{\rho}\right\}$. 
Lemma 3.10 ([21]). Assume that Conditions (C1)-(C2) hold and let $\left(w_{n}\right)$ be any sequence generated by (3.2), we have

$$
\left\|w_{n}-\bar{x}\right\|^{2} \leq\left\|x_{n}-\bar{x}\right\|^{2}-\left(1-\sigma^{2} \frac{\mu_{n}^{2}}{\mu_{n+1}^{2}}\right)\left\|x_{n}-v_{n}\right\|^{2}
$$

and

$$
\left\|w_{n}-v_{n}\right\| \leq \sigma \frac{\mu_{n}}{\mu_{n+1}}\left\|x_{n}-v_{n}\right\| .
$$

Lemma 3.11. Assume that Conditions (C1)-(C2) hold and suppose that

$$
\begin{array}{r}
\lim _{n \rightarrow \infty}\left\|x_{n}-u_{n}\right\|=\lim _{n \rightarrow \infty}\left\|x_{n}-v_{n}\right\|=\lim _{n \rightarrow \infty}\left\|x_{n}-w_{n}\right\|= \\
\lim _{n \rightarrow \infty}\left\|w_{n}-\left(\frac{1}{N} \sum_{i=0}^{N-1}\left(\left(1-\gamma_{n}\right) I d+\gamma_{n} S_{i}\right)\right) w_{n}\right\|=0 .
\end{array}
$$

Let $\left(x_{n}\right)$ and $\left(u_{n}\right)$ be two sequences generated by (3.2). If a subsequence $\left(x_{n_{t}}\right)$ of $x_{n}$ converges weakly to some $x^{*} \in \mathcal{H}$ then $x^{*} \in \Gamma$.

Proof. Let $x^{*} \in \mathcal{H}$ such that $x_{n_{t}} \rightarrow x^{*}$ then $x^{*} \in(A+B)^{-1}(0)$ follows from [21, Lemma 7]. Since $\lim _{n \rightarrow \infty}\left\|x_{n}-w_{n}\right\|=0$ and $x_{n_{t}} \rightarrow x^{*}$ therefore we have $w_{n_{t}} \rightarrow x^{*}$. Since

$$
\lim _{n \rightarrow \infty}\left\|w_{n}-\left(\frac{1}{N} \sum_{i=0}^{N-1}\left(\left(1-\gamma_{n}\right) I d+\gamma_{n} S_{i}\right)\right) w_{n}\right\|=0,
$$

therefore, utilizing Lemma 2.1, we get $x^{*} \in \operatorname{Fix}\left(S_{i}\right)$ and hence $x^{*} \in \Gamma$.

Now we are able to prove the main result of this section.

Proof of Theorem 3.2. For simplicity, the proof is divided into the following steps.

Step 1. Show that the sequence $\left(x_{n}\right)$ is bounded.

Let $\bar{x} \in \Gamma$, then for each $n \in \mathbb{N}$ we have

$$
\begin{aligned}
\left\|u_{n}-\bar{x}\right\|^{2} & =\left\|x_{n}-\bar{x}+\xi_{n}\left(x_{n}-x_{n-1}\right)\right\|^{2} \\
& \leq\left\|x_{n}-\bar{x}\right\|^{2}+\xi_{n}^{2}\left\|x_{n}-x_{n-1}\right\|^{2}+2 \xi_{n}\left\langle x_{n}-\bar{x}, x_{n}-x_{n-1}\right\rangle .
\end{aligned}
$$

Now for all $i \in\{1,2, \cdots, N\}$, set $S_{n}=\frac{1}{N} \sum_{i=0}^{N-1}\left(\left(1-\gamma_{n}\right) I d+\gamma_{n} S_{i}\right)$. Utilizing Lemma 2.3 for any $\bar{x} \in \Gamma$, we observe that

$$
\begin{aligned}
\left\|S_{n} x-\bar{x}\right\| & =\left\|\frac{1}{N} \sum_{i=0}^{N-1}\left(\left(1-\gamma_{n}\right) I d+\gamma_{n} S_{i}\right) x-\bar{x}\right\| \\
& \leq \frac{1}{N} \sum_{i=0}^{N-1}\left\|\left(\left(1-\gamma_{n}\right) I d+\gamma_{n} S_{i}\right) x-\bar{x}\right\| \\
& \leq \frac{1}{N} \sum_{i=0}^{N-1}\|x-\bar{x}\|=\|x-\bar{x}\| .
\end{aligned}
$$

It follows from the above estimate that $S_{n}$ is a quasi-nonexpansive operator. Since $\lim _{n \rightarrow \infty}(1-$ $\left.\sigma^{2} \frac{\mu_{n}^{2}}{\mu_{n+1}^{2}}\right)=1-\sigma^{2}>0$, therefore for each $n \geq n_{0}$ where $n_{0} \in \mathbb{N}$, we have that

$$
1-\sigma^{2} \frac{\mu_{n}^{2}}{\mu_{n+1}^{2}}>0 .
$$

From (3.3) and (3.6), we obtain

$$
\left\|w_{n}-\bar{x}\right\| \leq\left\|x_{n}-\bar{x}\right\|
$$


Further, from (C2) and (3.7), we have

$$
\begin{aligned}
\left\|x_{n+1}-\bar{x}\right\| \leq & \left\|\alpha_{n}\left(h\left(x_{n}\right)-\bar{x}\right)+\left(1-\alpha_{n}-\beta_{n}\right)\left(x_{n}-\bar{x}\right)+\beta_{n}\left(S_{n} w_{n}-\bar{x}\right)\right\| \\
\leq & \alpha_{n}\left\|h\left(x_{n}\right)-\bar{x}\right\|+\left(1-\alpha_{n}-\beta_{n}\right)\left\|x_{n}-\bar{x}\right\|+\beta_{n}\left\|S_{n} w_{n}-\bar{x}\right\| \\
\leq & \alpha_{n}\left\|h\left(x_{n}\right)-h(\bar{x})\right\|+\alpha_{n}\|h(\bar{x}-\bar{x})\|+\left(1-\alpha_{n}-\beta_{n}\right)\left\|x_{n}-\bar{x}\right\| \\
& +\beta_{n}\left\|w_{n}-\bar{x}\right\| \\
\leq & \alpha_{n} \lambda\left\|x_{n}-\bar{x}\right\|+\alpha_{n}\|h(\bar{x})-\bar{x}\|+\left(1-\alpha_{n}\right)\left\|x_{n}-\bar{x}\right\| \\
= & \left(1-\alpha_{n}(1-\lambda)\right)\left\|x_{n}-\bar{x}\right\|+\alpha_{n}(1-\lambda) \frac{\|h(\bar{x})-\bar{x}\|}{1-\lambda} \\
\leq & \max \left\{\left\|x_{n}-\bar{x}\right\|, \frac{\|h(\bar{x})-\bar{x}\|}{1-\lambda}\right\} .
\end{aligned}
$$

Thus, for all $n \geq n_{0},\left\|x_{n+1}-\bar{x}\right\| \leq \max \left\{\left\|x_{n_{0}}-\bar{x}\right\|, \frac{\|h(\bar{x})-\bar{x}\|}{1-\lambda}\right\}$. This implies that $\left(\left\|x_{n}-\bar{x}\right\|\right)$ is bounded.

Step 2. Compute the following two estimates:

$$
\begin{gathered}
(i): \beta_{n}\left(1-\sigma^{2} \frac{\mu_{n}^{2}}{\mu_{n+1}^{2}}\right)\left\|x_{n}-v_{n}\right\|^{2}+\beta_{n}\left(1-\alpha_{n}-\beta_{n}\right)\left\|x_{n}-S_{n} w_{n}\right\|^{2} \leq \\
\left\|x_{n}-\bar{x}\right\|^{2}-\left\|x_{n+1}-\bar{x}\right\|^{2}+\alpha_{n}\left\|h\left(x_{n}\right)-\bar{x}\right\|^{2}
\end{gathered}
$$

(ii) : $\left\|x_{n+1}-\bar{x}\right\|^{2} \leq\left[1-\alpha_{n}(1-\lambda)\right]\left\|x_{n}-\bar{x}\right\|^{2}$

$$
\left.+\alpha_{n}(1-\lambda)\left(\frac{2}{1-\lambda}\left(\beta_{n}\left\|x_{n}-S_{n} w_{n}\right\|\left\|x_{n+1}-\bar{x}\right\|+\left\langle h(\bar{x})-\bar{x}, x_{n+1}-\bar{x}\right)\right\rangle\right)\right) .
$$

\section{Utilizing Lemma 2.1(iii), we obtain}

$$
\begin{aligned}
\left\|x_{n+1}-\bar{x}\right\|^{2}= & \left\|\alpha_{n}\left(h\left(x_{n}\right)-\bar{x}\right)+\left(1-\alpha_{n}-\beta_{n}\right)\left(x_{n}-\bar{x}\right)+\beta_{n}\left(S_{n} w_{n}-\bar{x}\right)\right\|^{2} \\
= & \alpha_{n}\left\|h\left(x_{n}\right)-\bar{x}\right\|^{2}+\left(1-\alpha_{n}+\beta_{n}\right)\left\|x_{n}-\bar{x}\right\|^{2}+\beta_{n}\left\|\left(S_{n} w_{n}-\bar{x}\right)\right\|^{2} \\
& -\alpha_{n}\left(1-\alpha_{n}-\beta_{n}\right)\left\|h\left(x_{n}\right)-x_{n}\right\|^{2} \\
& -\beta_{n}\left(1-\alpha_{n}-\beta_{n}\right)\left\|x_{n}-S_{n} w_{n}\right\|^{2}-\alpha_{n} \beta_{n}\left\|h\left(x_{n}\right)-S_{n} w_{n}\right\|^{2} \\
\leq & \alpha_{n}\left\|h\left(x_{n}\right)-\bar{x}\right\|^{2}+\left(1-\alpha_{n}-\beta_{n}\right)\left\|x_{n}-\bar{x}\right\|^{2}+\beta_{n}\left\|w_{n}-\bar{x}\right\|^{2} \\
& -\beta_{n}\left(1-\alpha_{n}-\beta_{n}\right)\left\|x_{n}-S_{n} w_{n}\right\|^{2} .
\end{aligned}
$$

Now utilizing (3.3) in the above estimate, we get

$$
\begin{aligned}
\left\|x_{n+1}-\bar{x}\right\|^{2} \leq & \alpha_{n}\left\|h\left(x_{n}\right)-\bar{x}\right\|^{2}+\left(1-\alpha_{n}\right)\left\|x_{n}-\bar{x}\right\|^{2}-\beta_{n}\left(1-\alpha_{n}-\beta_{n}\right)\left\|x_{n}-S_{n} w_{n}\right\|^{2} \\
& -\beta_{n}\left(1-\sigma^{2} \frac{\mu^{2}}{\mu_{n+1}^{2}}\right)\left\|x_{n}-v_{n}\right\|^{2} \\
\leq & \alpha_{n}\left\|h\left(x_{n}\right)-\bar{x}\right\|^{2}+\left\|x_{n}-\bar{x}\right\|^{2}-\beta_{n}\left(1-\alpha_{n}-\beta_{n}\right)\left\|x_{n}-S_{n} w_{n}\right\|^{2} \\
& -\beta_{n}\left(1-\sigma^{2} \frac{\mu^{2}}{\mu_{n+1}^{2}}\right)\left\|x_{n}-v_{n}\right\|^{2} .
\end{aligned}
$$

Simplifying the above estimate, we have the desired estimate (3.8). Next, by using (C2) and setting $j_{n}=\left(1-\beta_{n}\right) x_{n}+\beta_{n} S_{n} w_{n}$, we get

$$
\left\|j_{n}-\bar{x}\right\| \leq\left\|x_{n}-\bar{x}\right\|
$$

and

$$
\left\|x_{n}-j_{n}\right\|=\beta_{n}\left\|x_{n}-S_{n} w_{n}\right\|
$$


Utilizing (3.10), (3.11) and Lemma 2.1(ii)-(iii), the desired estimate (3.9) follows from the following calculation:

$$
\begin{aligned}
&\left\|x_{n+1}-\bar{x}\right\|^{2} \\
&=\left\|\left(1-\alpha_{n}\right)\left(j_{n}-\bar{x}\right)+\alpha_{n}\left(h\left(x_{n}\right)-h(\bar{x})\right)-\alpha_{n}\left(x_{n}-j_{n}\right)-\alpha_{n}(\bar{x}-h(\bar{x}))\right\|^{2} \\
& \leq\left\|\left(1-\alpha_{n}\right)\left(j_{n}-\bar{x}\right)+\alpha_{n}\left(h\left(x_{n}\right)-h(\bar{x})\right)\right\|^{2}-2 \alpha_{n}\left\langle x_{n}-j_{n}+\bar{x}-h(\bar{x}), x_{n+1}-\bar{x}\right\rangle \\
& \leq\left(1-\alpha_{n}\right)\left\|j_{n}-\bar{x}\right\|^{2}+\alpha_{n}\left\|h\left(x_{n}\right)-h(\bar{x})\right\|^{2}-2 \alpha_{n}\left\langle x_{n}-j_{n}+\bar{x}-h(\bar{x}), x_{n+1}-\bar{x}\right\rangle \\
& \leq\left(1-\alpha_{n}\right)\left\|x_{n}-\bar{x}\right\|^{2}+\alpha_{n} \lambda\left\|x_{n}-\bar{x}\right\|^{2}+2 \alpha_{n}\left\langle x_{n}-j_{n}, \bar{x}-x_{n+1}\right\rangle \\
&+2 \alpha_{n}\left\langle h(\bar{x})-\bar{x}, x_{n+1}-\bar{x}\right\rangle \\
& \leq\left(1-\alpha_{n}(1-\lambda)\right)\left\|x_{n}-\bar{x}\right\|^{2}+2 \alpha_{n}\left\|x_{n}-j_{n}\right\|\left\|x_{n+1}-\bar{x}\right\|+2 \alpha_{n}\left\langle h(\bar{x})-\bar{x}, x_{n+1}-\bar{x}\right\rangle \\
&=\left(1-\alpha_{n}(1-\lambda)\right)\left\|x_{n}-\bar{x}\right\|^{2}+2 \alpha_{n} \beta_{n}\left\|x_{n}-S_{n} w_{n}\right\|\left\|x_{n+1}-\bar{x}\right\| \\
&+2 \alpha_{n}\left\langle h(\bar{x})-\bar{x}, x_{n+1}-\bar{x}\right\rangle \\
&=\left(1-\alpha_{n}(1-\lambda)\right)\left\|x_{n}-\bar{x}\right\|^{2}+\alpha_{n}(1-\lambda)\left(\frac { 2 } { 1 - \lambda } \left(\beta_{n}\left\|x_{n}-S_{n} w_{n}\right\|\left\|x_{n+1}-\bar{x}\right\|\right.\right. \\
&\left.\left.+\left\langle h(\bar{x})-\bar{x}, x_{n+1}-\bar{x}\right\rangle\right)\right) .
\end{aligned}
$$

Step 3. Show that $\lim _{n \rightarrow \infty}\left\|x_{n}-\bar{x}\right\|=0$.

We consider the two possible cases on the sequence $\left(\left\|x_{n}-\bar{x}\right\|\right)$.

Case A For all $n \geq n_{0},\left\|x_{n+1}-\bar{x}\right\|^{2} \leq\left\|x_{n}-\bar{x}\right\|^{2}$ and $n_{0} \in \mathbb{N}$. This implies that $\lim _{n \rightarrow \infty} \| x_{n}-$ $\bar{x} \|$ exists. Since $\lim _{n \rightarrow \infty}\left(1-\sigma^{2} \frac{\mu_{n}^{2}}{\mu_{n+1}^{2}}\right)=1-\sigma^{2}>0$. By using (C2) and (3.8), we have

$$
\lim _{n \rightarrow \infty}\left\|x_{n}-v_{n}\right\|=\lim _{n \rightarrow \infty}\left\|x_{n}-S_{n} w_{n}\right\|=0 .
$$

From (3.4), we get

$$
\lim _{n \rightarrow \infty}\left\|w_{n}-v_{n}\right\|=0
$$

By the definition of $\left(u_{n}\right)$ and (C1), we have

$$
\lim _{n \rightarrow \infty}\left\|u_{n}-x_{n}\right\|=\lim _{n \rightarrow \infty} \xi_{n}\left\|x_{n}-x_{n-1}\right\|=0 .
$$

By using the triangle inequality, we obtain the following estimates:

$$
\begin{aligned}
\left\|u_{n}-v_{n}\right\| & \leq\left\|u_{n}-x_{n}\right\|+\left\|x_{n}-v_{n}\right\| \rightarrow 0, \text { as } n \rightarrow \infty ; \\
\left\|u_{n}-w_{n}\right\| & \leq\left\|u_{n}-v_{n}\right\|+\left\|v_{n}-w_{n}\right\| \rightarrow 0, \text { as } n \rightarrow \infty ; \\
\left\|x_{n}-w_{n}\right\| & \leq\left\|x_{n}-v_{n}\right\|+\left\|v_{n}-w_{n}\right\| \rightarrow 0, \text { as } n \rightarrow \infty ; \\
\left\|w_{n}-S_{n} w_{n}\right\| & \leq\left\|x_{n}-w_{n}\right\|+\left\|x_{n}-S_{n} w_{n}\right\| \rightarrow 0, \text { as } n \rightarrow \infty .
\end{aligned}
$$

By using Lemma 2.4, we have

$$
\limsup _{n \rightarrow \infty}\left\|S_{n} w_{n}-S\left(S_{n} w_{n}\right)\right\|=0 .
$$

Note that for all $n \in \mathbb{N}$, we get

$$
\begin{aligned}
\left\|x_{n+1}-x_{n}\right\| & \leq\left\|x_{n+1}-S_{n} w_{n}\right\|+\left\|x_{n}-S_{n} w_{n}\right\| \\
& \leq \alpha_{n}\left\|h\left(x_{n}\right)-x_{n}\right\|+\left(2-\beta_{n}\right)\left\|x_{n}-S_{n} w_{n}\right\| .
\end{aligned}
$$

From (3.12) and (C2), the estimate (3.16) implies that

$$
\lim _{n \rightarrow \infty}\left\|x_{n+1}-x_{n}\right\|=0 .
$$

Similarly, from (3.14), (3.17) and the following triangle inequality, we have

$$
\left\|x_{n+1}-u_{n}\right\| \leq\left\|x_{n+1}-x_{n}\right\|+\left\|x_{n}-u_{n}\right\| \rightarrow 0, \text { as } n \rightarrow \infty \text {. }
$$


Since $\left(x_{n}\right)$ is bounded, then there exists a subsequence $\left(x_{n_{t}}\right)$ of $\left(x_{n}\right)$ with $x_{n_{t}} \rightarrow x^{*} \in \mathcal{H}$. Now utilizing Lemma 3.11 we have $x^{*} \in \Gamma$.

By making use of the estimate (3.17), we get

$$
\begin{aligned}
\limsup _{n \rightarrow \infty}\left\langle h(\bar{x})-\bar{x}, x_{n+1}-\bar{x}\right\rangle \leq & \limsup _{n \rightarrow \infty}\left\langle h(\bar{x})-\bar{x}, x_{n+1}-x_{n}\right\rangle+ \\
& \limsup _{n \rightarrow \infty}\left\langle h(\bar{x})-\bar{x}, x_{n}-\bar{x}\right\rangle \leq 0 .
\end{aligned}
$$

From the estimate (3.18) and Lemma 2.7, we get $\lim _{n \rightarrow \infty}\left\|x_{n}-\bar{x}\right\|=0$.

Case B There exists a subsequence $\left(\left\|x_{n_{k}}-\bar{x}\right\|^{2}\right)$ of $\left(\left\|x_{n}-\bar{x}\right\|^{2}\right)$ such that $\left\|x_{n_{k}}-\bar{x}\right\|<$ $\left\|x_{n_{k}+1}-\bar{x}\right\|$ for all $k \in \mathbb{N}$.

It follows from Lemma 2.8 that there exists a nondecreasing sequence $\left(b_{m}\right) \in \mathbb{N}$ such that $\lim _{m \rightarrow \infty} b_{m}=\infty$, for all $m \in \mathbb{N}$ with the inequality $\left\|x_{b_{m}}-\bar{x}\right\|^{2} \leq\left\|x_{b_{m}+1}-\bar{x}\right\|^{2}$ holds. In a similar fashion from (3.8), we obtain

$$
\begin{aligned}
& \beta_{b_{m}}\left(1-\sigma^{2} \frac{\mu_{b_{m}}^{2}}{\mu_{b_{m}+1}^{2}}\right)\left\|x_{b_{m}}-v_{b_{m}}\right\|^{2}+\beta_{b_{m}}\left(1-\alpha_{b_{m}}-\beta_{b_{m}}\right)\left\|x_{b_{m}}-S_{b_{m}} w_{b_{m}}\right\|^{2} \\
& \quad \leq\left\|x_{b_{m}}-\bar{x}\right\|^{2}-\left\|x_{b_{m}+1}-\bar{x}\right\|^{2}+\alpha_{b_{m}}\left\|h\left(x_{b_{m}}\right)-\bar{x}\right\|^{2} \\
& \quad \leq \alpha_{b_{m}}\left\|h\left(x_{b_{m}}\right)-\bar{x}\right\|^{2} .
\end{aligned}
$$

Since $\lim _{n \rightarrow \infty} \alpha_{n}=0$, so we get

$$
\lim _{m \rightarrow \infty}\left\|x_{b_{m}}-v_{b_{m}}\right\|=\lim _{m \rightarrow \infty}\left\|x_{b_{m}}-S_{b_{m}} w_{b_{m}}\right\|=0 .
$$

Similarly from Case A, we have

$$
\limsup _{m \rightarrow \infty}\left\langle h(\bar{x})-\bar{x}, x_{b_{m}+1}-\bar{x}\right\rangle \leq 0 .
$$

Using (3.9) for $n \geq \max \left\{n^{*}, n_{0}\right\}$, we have the following estimate:

$$
\begin{aligned}
& \left\|x_{b_{m}+1}-\bar{x}\right\|^{2} \\
& \leq \quad\left(1-\alpha_{b_{m}}(1-\lambda)\right)\left\|x_{b_{m}}-\bar{x}\right\|^{2}+\alpha_{b_{m}}(1-\lambda)\left(\frac { 2 } { 1 - \lambda } \left(\beta_{b_{m}}\left\|x_{b_{m}}-S_{b_{m}} w_{b_{m}}\right\|\left\|x_{b_{m}+1}-\bar{x}\right\|\right.\right. \\
& \left.\left.\quad+\left\langle h(\bar{x})-\bar{x}, x_{b_{m}+1}-\bar{x}\right\rangle\right)\right) \\
& \quad \leq\left(1-\alpha_{b_{m}}(1-\lambda)\right)\left\|x_{b_{m}+1}-\bar{x}\right\|^{2}+\alpha_{b_{m}}(1-\lambda)\left(\frac { 2 } { 1 - \lambda } \left(\beta_{b_{m}}\left\|x_{b_{m}}-S_{b_{m}} w_{b_{m}}\right\|\left\|x_{b_{m}+1}-\bar{x}\right\|\right.\right. \\
& \left.\left.\quad+\left\langle h(\bar{x})-\bar{x}, x_{b_{m}+1}-\bar{x}\right\rangle\right)\right) .
\end{aligned}
$$

The above estimate yields that

$$
\begin{aligned}
& \left\|x_{b_{m}+1}-\bar{x}\right\|^{2} \\
& \quad \leq \frac{2}{1-\lambda}\left(\beta_{b_{m}}\left\|x_{b_{m}}-S_{b_{m}} w_{b_{m}}\right\|\left\|x_{b_{m}+1}-\bar{x}\right\|+\left\langle h(\bar{x})-\bar{x}, x_{b_{m}+1}-\bar{x}\right\rangle\right) .
\end{aligned}
$$

Therefore, $\lim \sup _{m \rightarrow \infty}\left\|x_{b_{m}}-\bar{x}\right\|^{2} \leq 0$. Therefore, $x_{n} \rightarrow \bar{x} \in \Gamma$ and this completes the proof.

Replacing viscosity iteration in sequence (3.2) by Halpern's one, we have the following iterates:

Theorem 3.3. Let $A: \mathcal{H}_{1} \rightarrow 2^{\mathcal{H}}$ be a maximally monotone operator and let $B: \mathcal{H} \rightarrow \mathcal{H}_{1}$ be a monotone and $\rho$-Lipschitz operator for some $\rho>0$ defined on a real Hilbert space $\mathcal{H}$. For all $i \in\{1,2, \cdots, N\}, S_{i}: \mathcal{H} \rightarrow \mathcal{H}$ be a finite family of $\eta$-demimetric operators with $\eta \in(-\infty, 1)$ such that $I d-S_{i}$ is demiclosed at the origin. Assume that $\Gamma=(A+B)^{-1}(0) \cap \bigcap_{i=1}^{N} F i x\left(S_{i}\right) \neq \emptyset$, 
$\left(\mu_{1}\right)>0, \sigma \in(0,1),\left(\xi_{n}\right) \subset[0,1)$, and $\left(\alpha_{n}\right),\left(\beta_{n}\right)$ are sequences in $(0,1)$. For given $p, x_{0}, x_{1} \in$ $\mathcal{H}$, let the iterative sequence $\left(x_{n}\right)$ be generated by

$$
\left\{\begin{array}{l}
u_{n}=x_{n}+\xi_{n}\left(x_{n}-x_{n-1}\right) \\
v_{n}=J_{\mu_{n}}^{A}\left(I d-\mu_{n} B\right) u_{n} \\
w_{n}=v_{n}-\mu_{n}\left(B v_{n}-B u_{n}\right) \\
x_{n+1}=\alpha_{n} p+\left(1-\alpha_{n}-\beta_{n}\right) x_{n}+\beta_{n}\left(\frac{1}{N} \sum_{i=0}^{N-1}\left(\left(1-\gamma_{n}\right) I d+\gamma_{n} S_{i}\right)\right) w_{n} .
\end{array}\right.
$$

Assume that the following step size rule

$$
\mu_{n+1}=\left\{\begin{array}{l}
\min \left\{\frac{\sigma\left\|u_{n}-v_{n}\right\|}{\left\|B u_{n}-B v_{n}\right\|}, \mu_{n}\right\}, \text { if } B u_{n}-B v_{n} \neq 0 \\
\mu_{n}, \text { otherwise. }
\end{array}\right.
$$

and the conditions hold:

(C1) $\sum_{n=1}^{\infty} \xi_{n}\left\|x_{n}-x_{n-1}\right\|<\infty$;

(C2) $\lim _{n \rightarrow \infty} \frac{\alpha_{n}}{\beta_{n}}=0, \lim _{n \rightarrow \infty}\left(1-\alpha_{n}-\beta_{n}\right)=0$ and $\sum_{n=1}^{\infty} \frac{\alpha_{n}}{\beta_{n}}=\infty$.

(C3) For each $n \in \mathbb{N}, 0<a^{*}<\liminf _{n \rightarrow \infty} \beta_{n} \leq \limsup _{n \rightarrow \infty} \beta_{n}<b^{*}<1-\alpha_{n}$, where $a^{*}, b^{*}$ be positive real numbers.

Then the sequence $\left(x_{n}\right)$ generated by (3.20) converge strongly to a point $\bar{x}=P_{\Gamma} \circ h(\bar{x})$.

Remark 3.1. In order to obtain the desired result, we have to assume a stopping criteria for (3.20) like that if $n>n_{\max }$ for some chosen sufficiently large number $n_{\max }$.

Proof. Observe that for each $n \geq 1$, arguing similarly as in the proof of Theorem 3.2 (Steps 1-3), we deduce that $\Gamma$ is well-defined, closed and bounded. Furthermore, the sequence $\left(x_{k}\right)$ is bounded and

$$
\lim _{n \rightarrow \infty}\left\|x_{n+1}-x_{n}\right\|=0 .
$$

Let $x_{n+1}=\alpha_{n} p+\left(1-\alpha_{n}-\beta_{n}\right) x_{n}+\beta_{n} S_{n} w_{n}$. An easy calculation along (3.20) and (C2)-(C3) implies that

$$
\left\|S_{n} w_{n}-x_{n}\right\| \leq \frac{1}{\beta_{n}}\left\|x_{n+1}-x_{n}\right\|+\frac{\alpha_{n}}{\beta_{n}}\left\|h(p)-x_{n}\right\|+\frac{1-\alpha_{n}-\beta_{n}}{\beta_{n}}\left\|p-x_{n}\right\| .
$$

Hence, the above estimate implies that

$$
\lim _{n \rightarrow \infty}\left\|S_{n} w_{n}-x_{n}\right\|=0 .
$$

The rest of the proof follows immediately from the proof of Theorem 3.2 and is therefore omitted.

Remark 3.2. The condition (C1) is easily applicable in numerical calculation since the value of $\left\|x_{n}-x_{n-1}\right\|$ is known before choosing $\left(\xi_{n}\right)$ which satisfies $0 \leq \xi_{n} \leq \widehat{\xi_{n}}$

$$
\widehat{\xi_{n}}=\left\{\begin{array}{l}
\min \left\{\frac{\Theta_{n}}{\left\|x_{n}-x_{n-1}\right\|}, \xi\right\} \text { if } x_{n} \neq x_{n-1} ; \\
\xi \quad \text { otherwise, }
\end{array}\right.
$$

where $\left(\Theta_{n}\right)$ is a positive sequence such that $\sum_{n=1}^{\infty} \Theta_{n}<\infty$ and $\xi \in[0,1)$.

\section{Applications}

In this section, we illustrate some theoretical results as an application of our main result in Section 3. 
Split Convex Feasibility Problem. Let $\mathcal{H}_{1}$ and $\mathcal{H}_{2}$ be two real Hilbert spaces and $\hbar$ : $\mathcal{H}_{1} \rightarrow \mathcal{H}_{2}$ be a bounded linear operator. Let $C$ and $Q$ be nonempty, closed and convex subsets of $\mathcal{H}_{1}$ and $\mathcal{H}_{2}$, respectively. The formalism $\bar{x} \in C$ such that $\hbar \bar{x} \in Q$ is referred as the split convex feasibility problem (SCFP) where as the set $\omega:=C \cap \hbar^{-1}(Q)=\{\bar{x} \in C$ : $\hbar \bar{x} \in Q\}$ denotes the corresponding solutions of SCFP.

In the sequel, we recall the indicator function $b_{C}$ associated with the set $C$ as

$$
b_{C}(\bar{x}):=\left\{\begin{array}{l}
0, \bar{x} \in C \\
\infty, \text { otherwise. }
\end{array}\right.
$$

It is well-known that the proximal operator of $b_{C}$ is the metric projection on $C$

$$
\operatorname{prox}_{b_{C}}=\operatorname{argmin}_{\bar{p} \in C}\|\bar{p}-\bar{x}\|=P_{C}(\bar{x}) .
$$

Setting $B(\bar{x})=\hbar^{*}\left(I d-P_{Q}\right) \hbar \bar{x}$, where $P_{Q}$ is the metric projection onto $Q$ and $A(\bar{x})=$ $\operatorname{prox}_{b_{C}}(\bar{x})=\partial_{b_{C}}(\bar{x})$ then the SCFP has the inclusion structure as defined in (1.1). Since $B$ is $\rho$-Lipschitz continuous, where $\rho=\|\hbar\|^{2}=1$ and $A$ is maximal monotone, (see [8]), therefore, we compute the SCFP from the following result:

Theorem 4.4. Let $\mathcal{H}_{1}$ and $\mathcal{H}_{2}$ be two real Hilbert spaces and let $\hbar: \mathcal{H}_{1} \rightarrow \mathcal{H}_{2}$ be a bounded linear operator. Let $C$ and $Q$ be nonempty, closed and convex subsets of $\mathcal{H}_{1}$ and $\mathcal{H}_{2}$, respectively. Assume that $\Gamma=\omega \cap \bigcap_{i=1}^{N}$ Fix $\left(S_{i}\right) \neq \emptyset$ and $\left(\xi_{n}\right)$ is a bounded real sequence. For given $x_{0}, x_{1} \in \mathcal{H}_{1}$, let the iterative sequence $\left(x_{n}\right)$ be generated by

$$
\left\{\begin{array}{l}
u_{n}=x_{n}+\xi_{n}\left(x_{n}-x_{n-1}\right) \\
v_{n}=P_{C}\left(I d-\mu_{n} \hbar^{*}\left(I d-P_{Q}\right) \hbar\right) u_{n} \\
w_{n}=v_{n}-\mu_{n}\left(\left(\hbar^{*}\left(I d-P_{Q}\right) \hbar\right) v_{n}-\left(\hbar^{*}\left(I d-P_{Q}\right) \hbar\right) u_{n}\right) \\
x_{n+1}=\alpha_{n} h\left(x_{n}\right)+\left(1-\alpha_{n}-\beta_{n}\right) x_{n}+\beta_{n}\left(\frac{1}{N} \sum_{i=0}^{N-1}\left(\left(1-\gamma_{n}\right) I d+\gamma_{n} S_{i}\right)\right) w_{n}
\end{array}\right.
$$

Assume that the following step size rule

$$
\mu_{n+1}=\left\{\begin{array}{l}
\min \left\{\frac{\sigma\left\|u_{n}-v_{n}\right\|}{\left\|\left(\hbar^{*}\left(I d-P_{Q}\right) \hbar\right) u_{n}-\left(\hbar^{*}\left(I d-P_{Q}\right) \hbar\right) v_{n}\right\|}, \mu_{n}\right\} \\
\text { if }\left(\hbar^{*}\left(I d-P_{Q}\right) \hbar\right) u_{n}-\left(\hbar^{*}\left(I d-P_{Q}\right) \hbar\right) v_{n} \neq 0 \\
\mu_{n}, \text { otherwise, }
\end{array}\right.
$$

and the conditions (C1)-(C2) hold. Then the sequence $\left(x_{n}\right)$ generated by (4.22) converges strongly to an element $\bar{x}=P_{\Gamma} \circ h(\bar{x})$.

Convex Minimization Problems. Let $\mathfrak{f}: \mathcal{H} \rightarrow \mathbb{R}$ be a convex differentiable function and $\mathfrak{g}: \mathcal{H} \rightarrow \mathbb{R}$ be a convex lower semicontinuous function defined on a real Hilbert space $\mathcal{H}$. We consider the following convex minimization problem of finding $\bar{x} \in \mathcal{H}$ such that

$$
\mathfrak{f}(\bar{x})+\mathfrak{g}(\bar{x})=\min _{x \in \mathcal{H}}\{\mathfrak{f}(x)+\mathfrak{g}(x)\} .
$$

In view of the Fermat's rule, the problem (4.23) is equivalent to the following problem of finding $\bar{x} \in \mathcal{H}$ such that

$$
0 \in \nabla \mathfrak{f}(\bar{x})+\partial \mathfrak{g}(\bar{x}),
$$

where the subdifferential $\partial \mathfrak{g}$ is a maximal monotone operator and the gradient $\nabla \mathfrak{f}$ is $\rho$ Lipschitz continuous [8, 32]. Assume that $\omega$, the set of solutions of problem (4.23), is nonempty and setting $B:=\nabla \mathfrak{f}$ and $A:=\partial \mathfrak{g}$ in Theorem 3.2, we compute the following result:

Theorem 4.5. Let $\mathfrak{f}: \mathcal{H} \rightarrow \mathbb{R}$ be a convex differentiable function such that the gradient $\nabla \mathfrak{f}$ is $\rho$ Lipschitz continuous and $\mathfrak{g}: \mathcal{H} \rightarrow \mathbb{R}$ be a convex lower semicontinuous function defined on a real 
Hilbert space $\mathcal{H}$. Assume that $\Gamma=\omega \cap \bigcap_{i=1}^{N}$ Fix $\left(S_{i}\right) \neq \emptyset$ and $\left(\xi_{n}\right)$ is a bounded real sequence. For given $x_{0}, x_{1} \in \mathcal{H}$, let the iterative sequences $\left(x_{n}\right)$ be generated by

$$
\left\{\begin{array}{l}
u_{n}=x_{n}+\xi_{n}\left(x_{n}-x_{n-1}\right) \\
v_{n}=J_{\mu_{n}}^{\partial \mathfrak{g}}\left(I d-\mu_{n} \nabla \mathfrak{f}\right) u_{n} \\
w_{n}=v_{n}-\mu_{n}\left(\nabla \mathfrak{f} v_{n}-\nabla \mathfrak{f} u_{n}\right) \\
x_{n+1}=\alpha_{n} h\left(x_{n}\right)+\left(1-\alpha_{n}-\beta_{n}\right) x_{n}+\beta_{n}\left(\frac{1}{N} \sum_{i=0}^{N-1}\left(\left(1-\gamma_{n}\right) I d+\gamma_{n} S_{i}\right)\right) w_{n}
\end{array}\right.
$$

Assume that the following step size rule

$$
\mu_{n+1}=\left\{\begin{array}{l}
\min \left\{\frac{\sigma\left\|u_{n}-v_{n}\right\|}{\left\|\nabla \mathfrak{f} u_{n}-\nabla \mathfrak{f} v_{n}\right\|}, \mu_{n}\right\}, \text { if } \nabla \mathfrak{f} u_{n}-\nabla \mathfrak{f} v_{n} \neq 0 ; \\
\mu_{n}, \text { otherwise, }
\end{array}\right.
$$

and the conditions (C1)-(C2) hold. Then the sequence $\left(x_{n}\right)$ generated by (4.25) converges strongly to an element $\bar{x}=P_{\Gamma} \circ h(\bar{x})$.

Application to Image Processing Problems: Images are a main source of human information about the world. The theory of image processing deals with the restoration and enhancement of the original noisy and blurred images. For a given matrix $\hbar \in \mathbb{R}^{n \times n}$ describing a blur operator and a given vector $w \in \mathbb{R}^{n}$ representing the blurred and noisy image, the task is to estimate the unknown original image $z \in \mathbb{R}^{n}$ via the following convex minimization problem:

$$
\min _{z \in \mathbb{R}^{n}}\left\{\frac{1}{2}\|\hbar z-w\|_{2}^{2}+\mathbb{k}\|z\|_{1}\right\},
$$

where $\mathbb{k}>0$ is a regularization parameter.

In connection with Theorem 4.4, we set $A(z)=\|z\|_{1}, B(z)=\frac{1}{2}\|\hbar z-w\|_{2}^{2}$ and $\mathbb{k}=$ 0.7875. Also fix $\mu=0.001, \xi_{n}=\frac{1}{(100 * n+1)^{2}}, \alpha_{n}=\frac{1}{n}, \beta_{n}=\frac{1}{58 * n+1}$. The quality of the the restored images are analyzed on the following scale of signal to noise ratio (SNR) defined as $S N R=20 \log _{10} \frac{\|z\|^{2}}{\left\|z-z_{n}\right\|^{2}}$, where $z$ and $z_{n}$ are the original and estimated images at iteration $n$, respectively. We compare the performance of the algorithms abbreviated as Thm. 4.4, $\xi_{n} \neq 0$, Thm. $4.4, \xi_{n}=0$ and Theorem 2 of Gibali et. al [21] on the test images (Mona Lisa and Cameraman) via the image restoration experiment for motion operator, respectively.

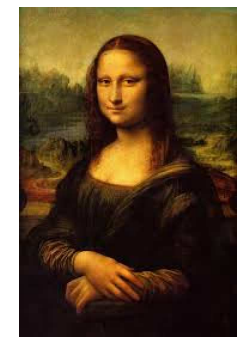

(A) Original image

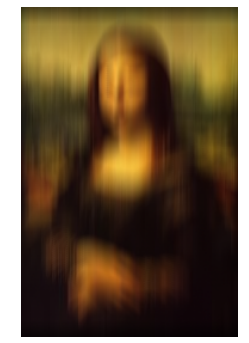

(в) Blurred and noisy image

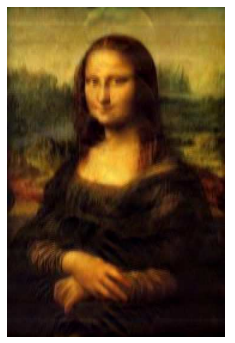

(c) Reconstructed image

FIGURE 1. (A) Original image $(182 \times 276)$ with a motion length 21 and an angle 31 (B) Blurred and noisy image, degraded by motion (C) Reconstructed image 


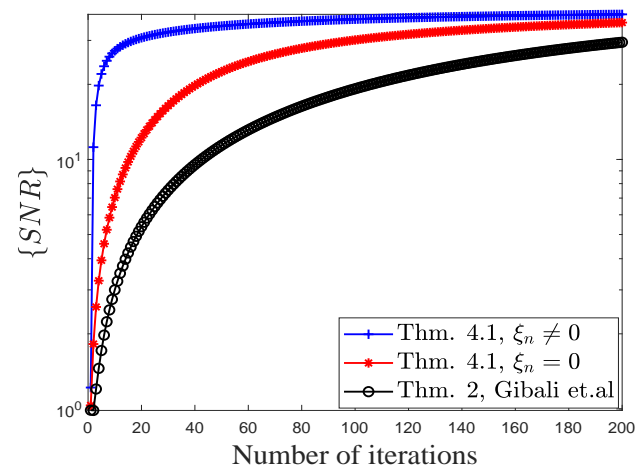

FIGURE 2. Comparison of Thm. 4.4, $\xi_{n} \neq 0$, Thm. $4.4, \xi_{n}=0$ and Theorem 2 of Gibali and Thong [21]

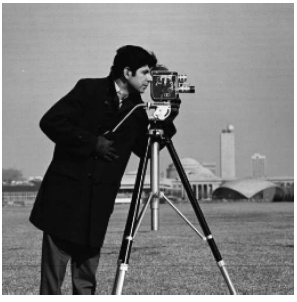

(A) Original image

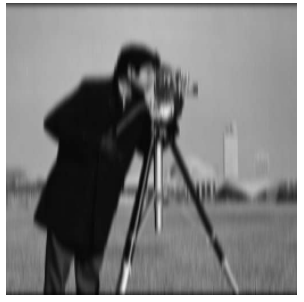

(B) Blurred and noisy image

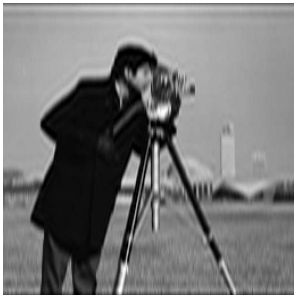

(C) Reconstructed image

FIGURE 3. (A) Original image $(256 \times 256)$ with Gaussian blur of size $9 \times 9$ and standard deviation $\sigma=4$ (B) Blurred and noisy image, degraded by Gaussian (C) Reconstructed image

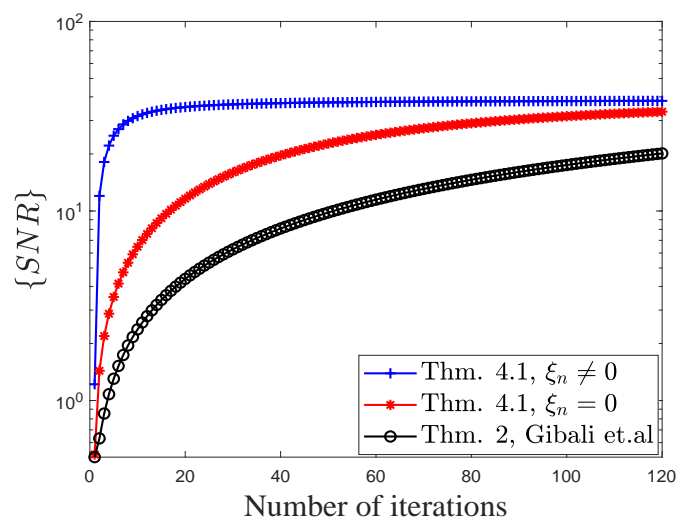

FIgURE 4. Comparison of Thm. 4.4, $\xi_{n} \neq 0$, Thm. $4.4, \xi_{n}=0$ and Theorem 2 of Gibali and Thong [21] 
TABLE 1. The SNR in decibel(dB) values and average per iteration computation time of the two optimization algorithms

\begin{tabular}{|l|l|l|l|l|}
\hline Algorithms & \multicolumn{2}{|c|}{ Mona Lisa } & \multicolumn{2}{c|}{ Cameraman } \\
\hline & SNR & CPU(sec) & SNR & CPU(sec) \\
\hline 1.Thm. 4.22, $\xi_{n} \neq 0$ & 38.249952 & 12.943470 & 38.148891 & 10.433934 \\
2.Thm. 4.22, $\xi_{n}=0$ & 35.364615 & 13.002959 & 36.732162 & 15.617105 \\
3.Thm. 2 of Gibali and Thong & 29.491617 & 17.632131 & 28.380412 & 20.112824 \\
\hline
\end{tabular}

It can be observed from Figure 2 and Figure 4 that the larger SNR values infer the better restored images. We can see from Table 1 that the Theorem 4.4 with $\xi_{n} \neq 0$ performs better as compared to the Theorem 4.4 with $\xi_{n}=0$ and Theorem 2 of Gibali and Thong [21].

\section{EXAMPLE AND NUMERICAL RESUlTS}

This section shows effectiveness to our algorithm by following given examples and numerical results.

Example 5.1. Let $\mathcal{H}=\mathbb{R}$, the set of all real numbers, with the inner product defined by $\langle x, y\rangle=x y$, for all $x, y \in \mathbb{R}$ and induced usual norm $|\cdot|$. For $\mu>0$, we define three operators $h, A, B: \mathbb{R} \rightarrow \mathbb{R}$ as $h(x)=\frac{x}{8}, A x=4 x$ and $B x=3 x$ for all $x \in \mathbb{R}$. For each $i \in\{1,2, \cdots, N\}$, let the operator $S_{i}: \mathbb{R} \rightarrow \mathbb{R}$ be defined by

$$
S_{i}(x)=\left\{\begin{array}{c}
-\frac{3 x}{i}, x \in(-\infty, 1) ; \\
x, x \in(1, \infty) .
\end{array}\right.
$$

For all $x=x_{0}, x_{1} \in \mathbb{R}$, then there exists unique sequence $\left(x_{n}\right)$ generated by the iterative method (3.2) converges strongly to a point in $P_{\Gamma} \circ h(\bar{x})$.

Now, observe that, $h: \mathcal{H} \rightarrow \mathcal{H}$ is a contraction operator with constant $\lambda \in[0,1), B$ a monotone and $\rho$-Lipschitz operator for some $\rho>0$ and $A$ a maximal monotone operator such that $(A+B)^{-1}(0)=\{0\}$. Note that $S_{i}$ is a finite family of $\frac{3-i^{2}}{(3+i)^{2}}$-demimetric operators with $\bigcap_{i=1}^{N} \operatorname{Fix}\left(S_{i}\right)=\{0\}$. Hence $\Gamma=(A+B)^{-1}(0) \cap \bigcap_{i=1}^{N} \operatorname{Fix}\left(S_{i}\right)(0)=0$.

In order to compute the numerical values of $\left(x_{n}\right)$, we choose $\Theta=0.5, \alpha_{n}=\frac{1}{n}, \beta_{n}=\frac{n}{2(n+1)}$, $\mu_{1}=7.45, \sigma=0.785$.

Since $\begin{cases}\min \left\{\frac{1}{n^{2}\left\|x_{n}-x_{n-1}\right\|}, 0.5\right\} \quad \text { if } \quad x_{n} \neq x_{n-1} & \text { otherwise. }\end{cases}$

Now, we provide a numerical test for a comparison between our accelerated Tseng type splitting method defined in (3.2) (i.e Thm. 3.2, $\xi_{n} \neq 0$ ), Tseng type splitting method (i.e Thm. 3.2, $\xi_{n}=0$ ) and Theorem 2 of Gibali and Thong [21]. The stopping criteria is defined as $E_{n}=\left\|x_{n}-x_{n-1}\right\|<10^{-5}$. The values of the sequence (3.2) in these cases have been computed for different choices of $x_{0}$ and $x_{1}$ in the following table:

TABLE 2. Numerical results for Example 5.1

\begin{tabular}{lllcccc}
\hline & Thm.3.1, & $\xi_{n} \neq 0$ & Thm.3.1, & $\xi_{n}=0$ & Thm. 2[21] & \\
\hline No. of Choices & Iter. & CPU(s) & Iter. & CPU(s) & Iter. & CPU(s) \\
$1 . x_{0}=3, x_{1}=2$ & 13 & 0.057912 & 17 & 0.061491 & 33 & 0.071583 \\
$2 . x_{0}=-6, x_{1}=4$ & 14 & 0.050666 & 20 & 0.054804 & 35 & 0.061804 \\
$3 . x_{0}=-1.7, x_{1}=-8.3$ & 15 & 0.051221 & 22 & 0.055838 & 37 & 0.062315 \\
\hline
\end{tabular}




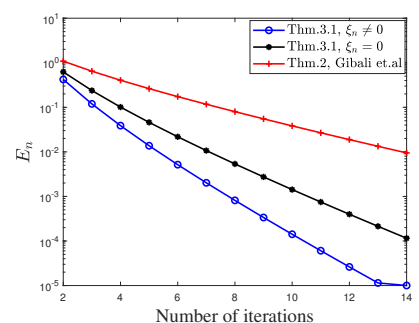

(A) Choice 1

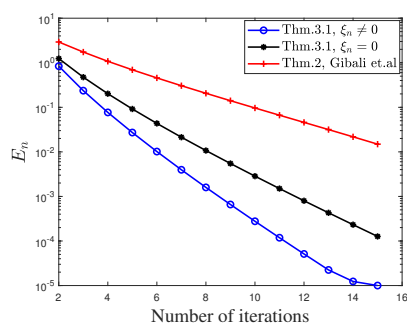

(B) Choice 2

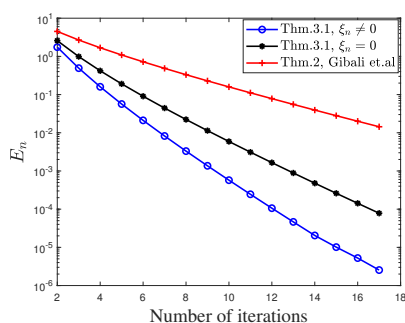

(c) Choice 3

FIGURE 5. Comparison of Thm. 3.2, $\xi_{n} \neq 0, \xi_{n}=0$ and Theorem 2 of Gibali and Thong

Now, we provide the numerical example for Theorem 4.4 to solve the SCFP in an infinite dimensional space $L_{2}([0,2 \pi])$.

Example 5.2. Let $\mathcal{H}_{1}=L^{2}([0,2 \pi])=\mathcal{H}_{2}$ with induced norm $\|x\|=\left(\int_{0}^{2 \pi}|x(s)|^{2} d s\right)^{\frac{1}{2}}$ and inner product $\langle x, y\rangle=\int_{0}^{2 \pi} x(s) y(s) d s$, for all $x, y \in L^{2}([0,2 \pi])$. The feasible set $C$ and $Q$ are given by: $C=\left\{x \in\left\{x \in \mathcal{H}_{1}: \int_{0}^{2 \pi} x(s) d s \leq 1\right\}\right.$ and now let the closed ball centered at $\sin \in L^{2}\left([0,2 \pi]\right.$ with radius 4 , that $Q=\left\{x \in\left\{x \in \mathcal{H}_{2}: \int_{0}^{2 \pi}|x(s)-\sin (s)|^{2} d s \leq 16\right\}\right.$. Let $\hbar: L^{2}([0,2 \pi]) \rightarrow L^{2}([0,2 \pi])$ be a bounded linear operator such that $(\hbar x)(s)=x(s)$, for all $x \in L^{2}([0,2 \pi])$. Then $\left(\hbar^{*} x\right)(s)=x(s)$ and $\|\hbar\|=1$. Further, $S_{i}=S$ for each $i=1,2, \cdots, N$, then the operator $S: \mathcal{H}_{1} \rightarrow \mathcal{H}_{1}$ is defined by

$$
S(x)=P_{C}(x)=\left\{\begin{array}{c}
\frac{x}{\|x\|},\|x\|>1 ; \\
x,\|x\| \leq 1 .
\end{array}\right.
$$

Consider the following problem:

$$
\text { Find } \bar{x} \in \Gamma=\omega \cap \operatorname{Fix}(S) \neq \emptyset \text {. }
$$

It is noted that $\omega$ is the convex feasibility problem is a problem of finding a point $\bar{x} \in \mathcal{H}$ such that $\bar{x} \in C \cap Q$. It is clear that $S$ is an $\eta$-demimetric operator and $h: \mathcal{H}_{1} \rightarrow \mathcal{H}_{2}$ is a contraction operator with constant $\lambda \in[0,1)$ defined as $h(x)(s)=\frac{x(s)}{8}$ for all $x \in$ $L^{2}([0,2 \pi]), s \in[0,2 \pi]$. Hence $\omega \cap F i x(S)=0$. Choose $\alpha_{n}=\frac{1}{15 \times n}$. The values of the Thm. 4.4 with $\xi_{n} \neq 0$, Thm. 4.4 with $\xi_{n}=0$ and Thm. 2 of Gibali and Thong [21] have been computed for different choices of $x_{0}(s)$ and $x_{1}(s), s \in[0,2 \pi]$ in the following table:

Choice 1. Choose $x_{0}=4\left(s^{2}-2 s\right) e^{2 s}+2 e^{4 s}, x_{1}=\frac{e^{t}}{\sin (s)}$

Choice 2. Choose $x_{0}=\left(s^{3}-3 s\right) \cos (4 s)+3 e^{2 s}, x_{1}=\left(s^{2}-e^{s}\right) \cos (s)$

Choice 3. Choose $x_{0}=\frac{3 \sin (s)}{8}, x_{1}=2 e^{s} s^{5}$.

The tolerance plotting $\left(E_{n}\right)$ against the Thm. 3.2-4.4 with $\xi_{n} \neq 0$, Thm. 3.2-4.4 with $\xi_{n}=0$ and Thm. 2 of Gibali and Thong [21] for each choices in Tables 2-3 and has shown in Figures 5-6.

We can see from Tables 2-3 and Figures 5-6, that the Thm. 3.2-4.4 with $\xi_{n} \neq 0$ performs better as compared to the Thm. 3.2-4.4 with $\xi_{n}=0$ and Thm. 2 of Gibali and Thong [21]. Elaborating the behaviour of these algorithms with respect to the Figures 5-6, the number 
TABLE 3. Comparison of Theorem $4.4, \xi_{n} \neq 0$, Theorem $4.4, \xi_{n}=0$ and Theorem 2 of Gibali and Thong [21]).

\begin{tabular}{lllllll}
\hline & \multicolumn{2}{c}{ No. of Iterations } & \multicolumn{2}{c}{ CPU Time(Sec) } & \\
\cline { 2 - 5 } & Choice 1 & Choice 2 & Choice 3 & Choice 1 & Choice 2 & Choice 3 \\
\hline Thm. $4.4, \xi_{n} \neq 0$, & 20 & 13 & 9 & 3.83664 & 2.98754 & 2.35670 \\
Thm. $4.4, \xi_{n}=0$, & 29 & 21 & 17 & 4.38926 & 3.39264 & 2.79551 \\
Thm. 2 $[21]$ & 46 & 37 & 32 & 5.08027 & 4.01928 & 3.01132 \\
\hline
\end{tabular}

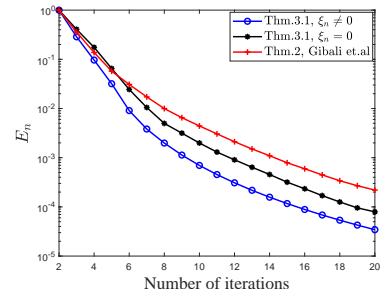

(A) Choice 1

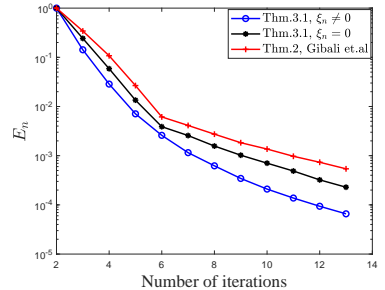

(B) Choice 2



(C) Choice 3

FIGURE 6. Comparison of Thm. $4.4, \xi_{n} \neq 0$, Thm. $4.4, \xi_{n}=0$ and Thm. 2 of Gibali and Thong [21]

of iterations required to converge to the common solution is expressed in Figures 5-6 (A, B, C). Summarizing these facts, we say that the Thm. 3.2, $\xi_{n} \neq 0$ exhibits a reduction in the tolerance, time and the number of iterations of the function as compared to the Thm. 3.2-4.4, $\xi_{n}=0$ and Thm. 2 of Gibali and Thong [21].

Conclusion. In this paper, we have devised an accelerated visco-Cesáro means Tseng type splitting method for computing a common solution of a monotone inclusion problems and the FPP associated with an $\eta$-demimetric operator in Hilbert spaces. The theoretical framework of the algorithm has been strengthened with an appropriate numerical example. Moreover, this framework has also been implemented to various instances of the inverse problems. We would like to emphasize that the above mentioned problems occur naturally in many applications like as mentioned above image processing to illustrate the convergence, therefore, iterative algorithms are inevitable in this field of investigation. As a consequence, our theoretical framework constitutes an important topic of future research.

Acknowledgment. The authors wish to thank the anonymous referees for their comments and suggestions. The authors (Y. Arfat, P. Kumam and P. S. Ngiamsunthorn) acknowledge the financial support provided by the Center of Excellence in Theoretical and Computational Science (TaCS-CoE), KMUTT. Moreover, this project is funded by National Council of Thailand (NRCT) under Research Grants for Talented Mid-Career Researchers (Contract no. N41A640089). The author Y. Arfat was supported by the Petchra Pra Jom Klao Ph.D Research Scholarship from King Mongkut's University of Technology Thonburi, Thailand(Grant No.16/2562).

Competing interests. The authors declare that they have no competing interests. 
Authors' contributions. All authors contributed equally and significantly in writing this article. All authors read and approved the final manuscript.

Availability of Data and Material. Data sharing not applicable to this article as no datasets were generated or analyzed during the current study.

\section{REFERENCES}

[1] Alvarez, F.; Attouch, H. An inertial proximal method for monotone operators via discretization of a nonlinear oscillator with damping. Set-Valued Anal. 9 (2001), 3-11.

[2] Arfat, Y.; Kumam. P.; Ngiamsunthorn. P. S. et al. An inertial based Forward-Backward algorithm for monotone inclusion problems and split mixed equilibrium problems in Hilbert spaces. Adv. Differ. Equ. 2020 (2020), 453.

[3] Arfat, Y.; Kumam, P.; Ngiamsunthorn, P. S. et al., Approximation results for split equilibrium problems and fixed point problems of nonexpansive semigroup in Hilbert spaces. Adv. Differ. Equ. 2020 (2020), 512.

[4] Arfat, Y.; Kumam, P.; Khan, M. A. A. et al., An inertially constructed forward-backward splitting algorithm in Hilbert spaces. Adv. Differ. Equ. 2021 (2021), 124.

[5] Arfat, Y.; Kumam, P.; Khan, M. A. A.; Ngiamsunthorn, P. S.; Kaewkhao, A. A parallel hybrid accelerated extragradient algorithm for pseudomonotone equilibrium, fixed point, and split null point problems. Adv. Differ. Equ. 2021 (2021), 364.

[6] Arfat, Y.; Kumam, P.; Ngiamsunthorn, P. S.; Khan, M. A. A. An accelerated projection based parallel hybrid algorithm for fixed point and split null point problems in Hilbert spaces. Math. Meth. Appl. Sci. (2021), 1-19. doi.org/10.1002/mma.7405.

[7] Arfat, Y.; Kumam, P.; Khan, M. A. A.; Ngiamsunthorn, P. S. Parallel shrinking inertial extragradient approximants for pseudomonotone equilibrium, fixed point and generalized split null point problem. Ricerche Mat. (2021). https://doi.org/10.1007/s11587-021-00647-4.

[8] Bauschke, H. H.; Combettes, P. L. Convex Analysis and Monotone Operators Theory in Hilbert Spaces. CMS Books in Mathematics, Springer, New York, (2011).

[9] Baillon, J. B. Un theorem de type ergodique pour les contractions non lineairs dans un e'spaces de Hilbert. C.R. Acad. Sci. Paris Ser., A-B 280 (1975), 1511-1541.

[10] Brézix, H. Chapitre, I. I. Operateurs maximaux monotones. North-Holland Math. Stud. 5 (1973), 19-51.

[11] Browder, F. E.; Petryshyn, W. V. Construction of fixed points of nonlinear mappings in Hilbert spaces. J. Math. Anal. Appl. 20 (1967), 197-228.

[12] Bruck, R. E. On the convex approximation property and the asymptotic behavior of Nonlinear contractions in Banach spaces. Israel J. Math. 38 (1981), 304-314.

[13] Byrne. C. A unified treatment of some iterative algorithms in signal processing and image reconstruction. Inverse Probl. 20 (2004), no. 1, 103-120.

[14] Censor, Y.; Elfving, T. A multiprojection algorithm using Bregman projections in a product space. Numer. Algorithms 8 (1994), 221-239.

[15] Combettes, P. L. The convex feasibility problem in image recovery. Adv. Imaging Electron Phys. 95 (1996), $155-453$.

[16] Deepho, J.; Moreno, J. M.; Sitthithakerngkiet, K.; Kumam, P. Convergence analysis of hybrid projection with Ces/' aro mean method for the split equilibrium and general system of finite variational inequalities. J. Comp. Appl. Math. 318 (2017), 658-673.

[17] Douglas, J.; Rachford, H. H. On the numerical solution of the heat conduction problem in two and three space variables. Trans. Amer. Math. Soc. 82 (1956), 421-439.

[18] Duchi, J.; Shalev-Shwartz, S.; Singer, Y.; Chandra, T. Efficient projections onto the 11-ball for learning in high dimensions. Proceedings of the 25 th International Conference on Machine Learning, Helsinki, Finland (2008).

[19] Engl, H. W.; Hanke, M.; Neubauer, A. Regularization of Inverse Problems. Kluwer Academic Publishers, Dordrecht, (2000).

[20] Genel, A.; Lindenstrauss, J. An example concerning fixed points. Israel J. Math. 22 (1975), 81-86.

[21] Gibali, A.; Thong, D. V. Tseng type methods for solving inclusion problems and its applications. Calcolo 55 (2018), 49.

[22] Gibali, A. A new split inverse problem and an application to least intensity feasible solutions. Pure. Appl. Func. Anal. 2 (2017), 243-258.

[23] Gibali, A.; Reich, S.; Zalas, R. Outer approximation methods for solving variational inequalities in Hilbert space. Optimization 66 (2017), 417-437.

[24] Halpern, B. Fixed points of nonexpansive maps. Bull. Amer. Math. Soc. 73 (1967), 957-961. 
[25] Harisa, S. A.; Khan, M. A. A.; Mumtaz, F.; Farid, N.; Morsy, A.; Nisar, K. S.; Ghaffar, A. Shrinking Cesáro means method for the split equilibrium and fixed point problems in Hilbert spaces. Adv. Differ. Equ. 2020 (2020), 345.

[26] Hirano, N.; Takahashi, W. Nonlinear ergodic theorems for nonexpansive mappings in Hilbert spaces. Kodai Math. J. 2 (1979), 11-25.

[27] Lions, P. L.; Mercier, B. Splitting algorithms for the sum of two nonlinear operators. SIAM J. Numer. Anal. 16 (1979), 964-979.

[28] Maingé, F. A hybrid extragradient-viscosity method for monotone operators and fixed point problems. SIAM J. Control Optim. 47 (2008), 1499-1515.

[29] Moudafi, A. Viscosity Approximation Methods for Fixed-Points Problems. J. Math. Anal. Appl. 241 (2000), No. 1, 46-55.

[30] Pasty, G. B. Ergodic Convergence to a Zero of the Sum of Monotone Operators in Hilbert Space. J. Math. Anal. Appl. 72 (1979), 383-390.

[31] Polyak, B. T. Introduction to Optimization, Optimization Software. New York (1987).

[32] Rockafellar, R. T. On the maximality of sums of nonlinear monotone operators. Trans. Amer. Math. Soc. 149 (1970), 75-88.

[33] Takahashi, W. The split common fixed point problem and the shrinking projection method in Banach spaces. J. Conv. Anal. 24 (2017), 1015-1028.

[34] Takahashi, W.; Wen, C. F.; Yao, J. C. The shrinking projection method for a finite family of demimetric mappings with variational inequality problems in a Hilbert space. Fixed Point Theory 19 (2018), 407-419.

[35] Tseng, P. A modified forward-backward splitting method for maximal monotone mappings. SIAM J. Control. Optim. 38 (2000), 431-446.

[36] Vogel, C. R. Computational Methods for Inverse Problems. SIAM, Philadelphia, (2002).

[37] Xu, H. K. Iterative algorithms for nonlinear operators. J. Lond. Math. Soc. 66 (2002), 240-256.

${ }^{1}$ KMUTT FIXED POINT RESEARCH LABORATORY

KMUTT-Fixed POINT THEORY AND APPLiCATIONS RESEARCH GROUP

DEPARTMENT OF MATHEMATICS

FACULTY OF SCIENCE

King MongKut's University of TeCHNOlOgy THONBURI (KMUTT)

126 Pracha-Uthit RoAD, BANG Mod, Thung KHRU, BANGKOK 10140, THAilAnd

Email address: yasir.arfat@mail.kmutt.ac.th

Email address: poom.kum@kmutt.ac.th

${ }^{2}$ Center of Excellence in Theoretical and Computational SCience (TaCS-CoE)

SCL 802 FIXED POINT LABORATORY

SCIENCE LABORATORY BUILDING

King MONGKUT'S UNIVERSITY OF TECHNOLOGY THONBURI (KMUTT)

126 Pracha-Uthit RoAd, BAng Mod, Thung KHrU, BANGKOK 10140, Thailand

Email address: poom. kumekmutt.ac.th

${ }^{3}$ Research CENTER FOR INTERNEURAL COMPUting

China Medical University Hospital

China Medical University, TAICHUNG, 40402, TAIWAN

Email address: poom.kumekmutt.ac.th

${ }^{4}$ DEPARTMENT OF MATHEMATICS

COMSATS UNIVERSITY ISLAMABAD

LAHORE CAMPUS, LAHORE 54000, PAKISTAN

Email address: itsakb@hotmail.com

${ }^{5}$ DEPARTMENT OF MATHEMATICS

FACULTY OF SCIENCE

King MONGKUT'S UNIVERSITY OF TECHNOLOGY THONBURI (KMUTT)

126 PRACHA-Uthit RoAd, BANG MOD, Thung KHRU, BANGKOK 10140, ThAILAND

Email address: parinya.san@kmutt.ac.th 